\title{
Diagnosis and Management of Primary Umbilical Melanoma with Omphalitis Features
}

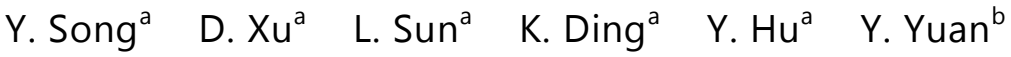 \\ Departments of ${ }^{a}$ Surgical Oncology and ${ }^{b}$ Medical Oncology, The Second Affiliated \\ Hospital, Zhejiang University College of Medicine, Hangzhou, China
}

\section{Key Words}

Melanoma $\cdot$ Umbilicus $\cdot$ Omphalitis

\begin{abstract}
We report on a 62-year-old Chinese male patient who presented with a pigmented lesion in the umbilical area and who was treated for omphalitis in a local hospital for 1 month. Biopsy pathological examination revealed that the lesion was a melanoma. The Breslow depth was estimated at $3 \mathrm{~mm}$, and Clark's level was IV. Ultrasonography showed no suspicious pathological lymph nodes, and no metastases were detected in the lung, liver or brain through CT scans. Reexcision was performed with lateral margins $>2 \mathrm{~cm}$ from the original scar down to the peritoneum, including the umbilicus, round ligament of the liver and urachus. No adjuvant therapy was suggested, and the patient was still alive without recurrences or metastases after 36 months.
\end{abstract}

\section{Background}

Melanoma is one of the most common cutaneous malignancies in the world. Over the past decades, melanoma-associated morbidity has been steadily increasing. Melanoma often occurs in sun-exposed areas of the skin in white populations, such as the upper back in men and the back and lower legs in women [1]. The most common site of cutaneous melanoma in non-white populations (such as the Chinese) is the feet [2]. However, melanoma arising in the umbilicus is extremely rare. Here, we report a case of primary umbilical melanoma, whose proper treatment was delayed for 1 month because of misdiagnosis. 
Song et al.: Diagnosis and Management of Primary Umbilical Melanoma with Omphalitis Features

\section{Case Presentation}

A 62-year-old Chinese male patient presented with a 1-month history of a pigmented lesion in the umbilical area. He noticed the brown nevus 4 years before examination. Until 1 month prior to examination, there was no obvious change of the nevus, but then it gradually grew larger and produced discharge. For 1 month, the patient was treated for omphalitis with antibiotics in a local hospital - without success. Subsequently, he was referred to the oncology outpatient clinic of our hospital. Physical examination revealed a brown lesion sized $2 \times 1 \mathrm{~cm}$ with an irregular border on the vertical sidewall of the umbilicus. Neither visible satellite lesions nor palpable superficial pathological lymph nodes were detected. Excision biopsy was performed with $1 \mathrm{~cm}$ margins beyond the border of the lesion. Pathological examination revealed that the lesion was a malignant melanoma. The Breslow depth was estimated at $3 \mathrm{~mm}$. Clark's level was IV (fig. 1a) and tumor mitotic rate $\geq 6$ mitoses $/ \mathrm{mm}^{2}$ (fig. 1b). Ultrasonography of the axillary and groin areas revealed no suspicious pathological lymph nodes, and no metastases were detected in the lung, liver or brain through CT scans. A wide, irregular reexcision was subsequently performed with lateral margins $>2 \mathrm{~cm}$ (fig. 2a) from the original scar down to the peritoneum, including the umbilicus, round ligament of the liver and urachus (fig. 2b). We concluded the procedure by closing the abdominal cavity and incision directly (fig. 2c). No adjuvant therapy was suggested after surgery excision, and the patient has remained disease-free in the 36 months since surgery.

\section{Discussion}

For several reasons, diagnosis of navel melanoma is usually delayed. First, the umbilicus is a hidden area, and therefore tumors are not easy to detect for patients and doctors, especially in overweight patients. Second, primary melanomas originating from the umbilicus are extremely rare, and doctors lack the knowledge of both diagnosis and treatment. In 1916, Cullen [3] reported 3 presumed cases of umbilical melanoma. Since then, only 13 cases have been reported in the literature. In 1965, Steck and Helwig [4] collected a large series of 112 umbilical tumors and reported 4 cases of primary melanomas. In addition to Steck and Helwig's work [4], only 9 other individual cases are reported in the literature [5-12]. About half of these cases developed from a preexisting nevus [4-12], which is similar to our case.

Our patient was misdiagnosed with omphalitis and treated with antibiotics for 1 month. Based on this experience, careful follow-up examinations are highly suggested in patients with umbilical nevus and omphalitis. If special changes such as an increase in size or diabrosis on the surface are observed, surgery excision (even a prophylactic excision) should be considered early to determine if the patient has umbilical melanoma, even if it appears only to be omphalitis [10].

The treatment of umbilical melanoma is also difficult because of the complex anatomy of the umbilicus [9]. After delivery, the umbilical cord retains some ligaments connecting the umbilicus to the peritoneum and intraabdominal visceral organs such as the bladder and liver. Accordingly, it is possible that umbilical melanoma grow into and along these fibrous remnant attachments. Local recurrence was reported due to remaining melanoma cell nests in the omphalomesenteric duct [9]. We performed a surgical excision down to the peritoneum, including the umbilicus, round ligament of the liver and urachus to eliminate possible melanoma cell remainings. 
Reconstruction of the navel region has been previously reported after surgical excision [13-15]. However, sometimes it is a difficult procedure to perform because of the wide excision necessary to remove the cancerous material, as was the case with our patient. Therefore, we used an irregular ' $\mathrm{T}$ '-shape incision, which can reduce the tension of the abdominal wall and allows easy placement of sutures within the abdominal cavity.

Due to its low incidence, the efficacy of sentinel lymph node biopsy in primary malignant umbilical melanoma is still ambiguous [12]. As no suspicious regional lymph node metastasis was detected with ultrasonography, sentinel lymph node biopsy was not routinely performed in our patient.

After a 36-month follow-up, there were no suspicious local recurrences or metastases to the regional lymph nodes, and ultrasound testing of the abdominal wall around the incision, axillary and groin lymph nodes was negative as well.

Primary umbilical melanoma is rare, and therefore surgeons should keep in mind that follow-up examinations of patients with umbilical nevus and omphalitis are very important. To treat an umbilical melanoma, clear, deep and lateral margins are necessary. Excision of the entire umbilicus, including its attachment to the peritoneum, is recommended, and in some cases, reconstruction of the navel region can be performed.

\section{Disclosure Statement}

The authors declare that they have no competing interests.

\section{References}

1 Hall HI, Miller DR, Rogers JD, Bewerse B: Update on the incidence and mortality from melanoma in the United States. J Am Acad Dermatol 1999;40:35-42.

2 Hui SK, Lau KH, Leung CY, Tang WYM, Yau KC, Trendell-Smith NJ, Wong TW, Chong LY, Lo KK: Cutaneous melanoma: clinical features of 32 Hong Kong Chinese patients. Hong Kong J Dermatol Venereol 2005;13:130-140.

3 Cullen TS: Embryology, Anatomy, and Diseases of the Umbilicus, together with Diseases of the Urachus. Philadelphia, W.B. Saunders, 1916, pp 457-458.

4 Steck WD, Helwig EB: Tumors of the umbilicus. Cancer 1965;18:907-915.

5 Hughes J: Melanoma of the umbilicus - a case report. J Ir Med Assoc 1963;53:94-96.

6 Barrow MV: Metastatic tumors of the umbilicus. J Chronic Dis 1966;19:1113-1117.

7 Charoenkul V, DelCampo A, Derby A, Hodgson WJ, McElhinney AJ: Tumors of the umbilicus. Mt Sinai J Med 1977;44:257-262.

$\checkmark 8$ Colonna MR, Giovannini UM, Sturniolo G, Colonna U: The umbilicus: a rare site for melanoma. Clinical considerations in two cases. Case reports. Scand J Plast Reconstr Surg Hand Surg 1999;33:449-452.

9 Meine JG, Bailin PL: Primary melanoma of the umbilicus: report of a case and review of the relevant anatomy. Dermatol Surg 2003;29:404-407.

10 Campos-Muñoz L, Quesada-Cortés A, Ruiz E, Casado M, Pizarro A: Primary melanoma of the umbilicus appearing as omphalitis. Clin Exp Dermatol 2007;32:322-324.

11 Mangas C, Romaní J, Muñoz C, Luelmo J: Navel melanoma: not always easy to detect, not always difficult to remove. Dermatol Online J 2008;14:20.

12 Cecchi R, Pavesi M, Buralli L, Rapicano V, De Gaudio C: Primary umbilical melanoma. Australas J Dermatol. 2009;50:220-222.

13 Breuninger H, Zimmermann C: Umbilical reconstruction after excision of melanomas in the area of the umbilicus (in German). Hautarzt 1996;47:273-275.

-14 Kakudo N, Kusumoto K, Fujimori S, Shimotsuma A, Ogawa Y: Reconstruction of a natural-appearing umbilicus using an island flap: case report. J Plast Reconstr Aesthet Surg 2006;59:999-1002.

15 Castillo PF, Sepúlveda CA, Prado AC, Troncoso AL, Villamán JJ: Umbilical reinsertion in abdominoplasty: technique using deepithelialized skinflaps. Aesthetic Plast Surg 2007;31:519-520. 


\section{Case Reports in Oncology}

\begin{tabular}{l|l}
\hline Case Rep Oncol 2013;6:154-157 & \\
\hline DOI: $\underline{10.1159 / 000350421}$ & $\begin{array}{l}\text { C 2013 S. Karger AG, Basel } \\
\text { www.karger.com/cro }\end{array}$ \\
\hline
\end{tabular}

Song et al.: Diagnosis and Management of Primary Umbilical Melanoma with Omphalitis Features
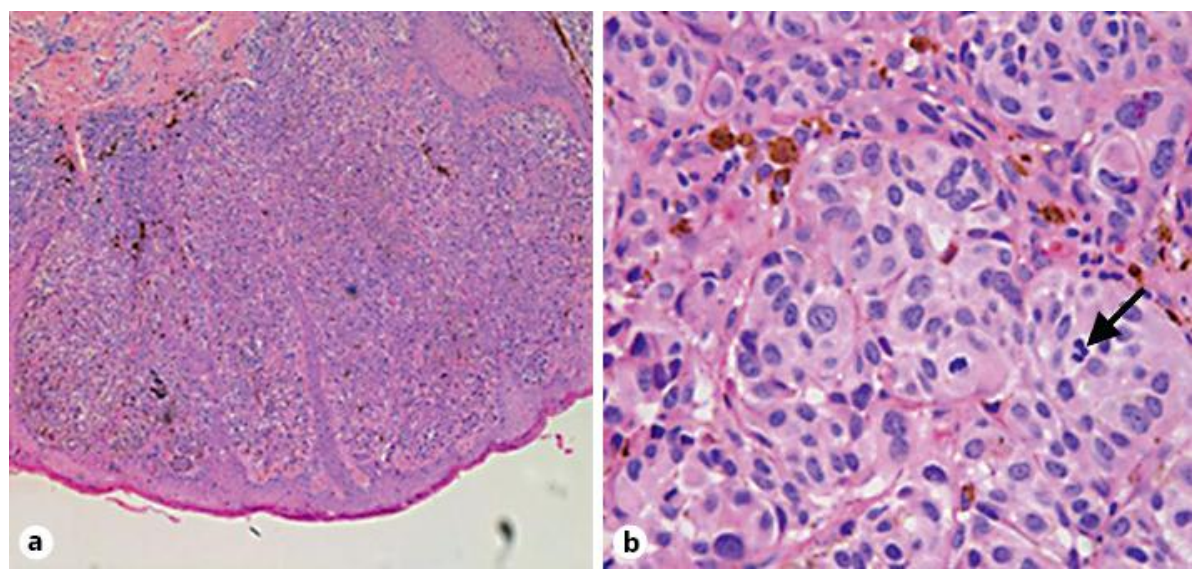

Fig. 1. a First biopsy from the umbilicus showing a malignant melanoma with a tumor thickness of $3 \mathrm{~mm}$ and Clark's level IV (HE. $\times 5$ ). $\mathbf{b}$ The arrow points to the mitosis of tumor cells (HE. $\times 40$ ).
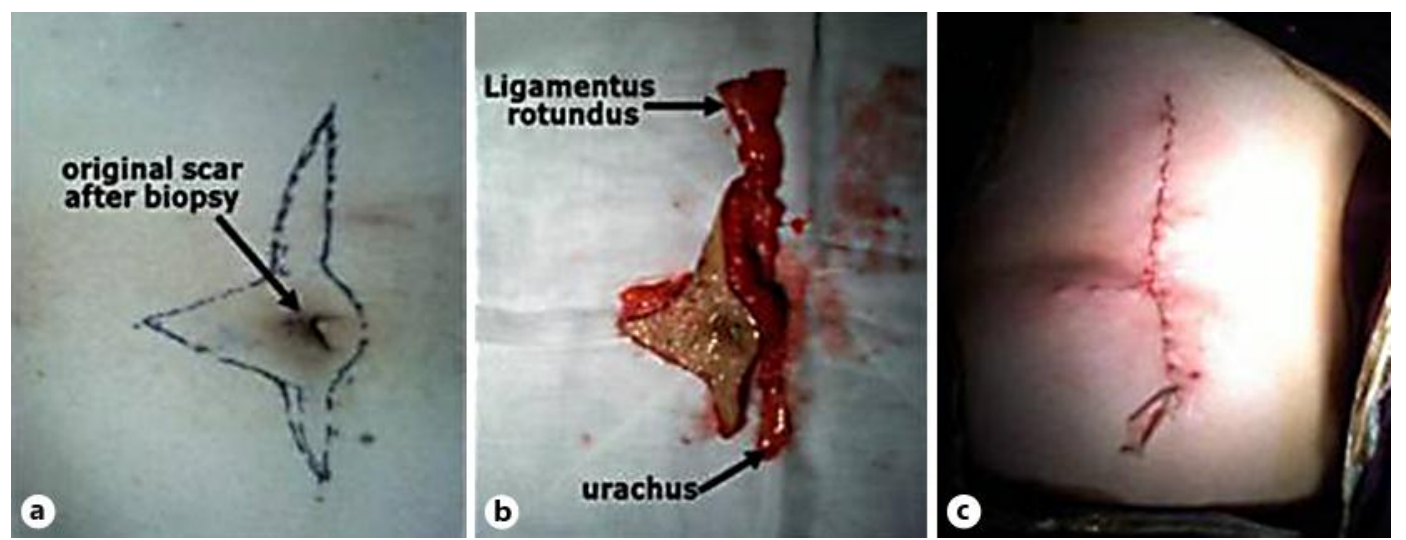

Fig. 2. a An irregular incision was made with lateral margins $>2 \mathrm{~cm}$ from the original scar (indicated by an arrow). $\mathbf{b}$ Gross view of the specimen, including the umbilicus, round ligament of the liver (ligamentus rotundus) and urachus. c Incision site after reconstruction. 